\title{
Comparison of Depolarization and Depolarization in Mathematical Models of the Left Ventricle and the Longitudinal Ventricular Slice
}

\author{
Anastasia E Bazhutina ${ }^{1}$, Anastasia Khokhlova ${ }^{1,2}$, Konstantin S Ushenin ${ }^{1,2}$, Olga Solovyova ${ }^{1,2}$ \\ ${ }^{1}$ Ural Federal University, \\ ${ }^{2}$ Institute of Immunology and Physiology, Ekaterinburg, Russia
}

\begin{abstract}
Myocardial slices are widely used for cardiac electrophysiology research but correspondence of electrophysiological properties between the cardiac slices and the whole heart has not been studied in details. The aim of this study is to investigate the differences in electrophysiological properties between the left ventricle and the longitudinal ventricular slice passing through the apex using mathematical models. ECG signals and the time of activation and repolarization, repolarization dispersion and dispersion of action potential duration were compared. We have shown that the electrophysiological processes in the ventricle and the longitudinal ventricular slice are quite similar, so we believe that cardiac slices can be used to evaluate global electrophysiological properties of the ventricles. The local differences obtained can be explained by differences in geometry and fiber orientation locally affecting depolarization and repolarization in the myocardium.
\end{abstract}

\section{Introduction}

Thin $(250-350 \mu \mathrm{m})$ myocardial slices are widely used in basic research as a balance between experimental models of single cardiomyocytes and whole hearts. In these slices cells are exposed to more realistic electrical and mechanical interactions than in the cardiac culture, so it is believed that this pseudo-2D model represents an organotypic model of the heart [1]. This experimental model is utilized to study cardiac electrophysiology and calcium imaging [2,3], mechanics [4] and energetics [5], chemical interventions [6] etc.

Previous studies have shown that conduction velocities in human and dog slices were similar to the values for the whole hearts [7]. However, the correspondence of the ECG signal and activation and repolarization maps between the cardiac slices and the whole hearts has not been studied. In this study we present preliminary results on the differences in of electrophysiological properties between the left ventricle (LV) and the longitudinal ventricular slice passing through the LV apex using mathematical models.

\section{Methods}

\subsection{Model geometry}

The realistic geometry with fiber orientations of the LV model was reconstructed using diffusion tensor magnetic resonance imaging (DT-MRI) data of the canine heart from the public database of the Johns Hopkins University.

The following algorithm was used to generate a tetrahedral 3D mesh of the whole LV model and a triangular 2D mesh of the LV slice model from DT-MRI data. A binary mask of the LV was created using morphological operations. A surface triangular mesh was built by cube marching algorithm. Then, a tetrahedral 3D mesh suitable for finite element simulation was generated. To generate a 2D mesh, the contour of the LV slice was reconstructed by intersection of the LV surface mesh with a plane passing through the LV apex. At the final step, the obtained LV slice was triangulated. An average element length was 0.7 $\mathrm{mm}$ for both tetrahedral and triangular meshes.

\subsection{Myocardial anisotropy}

Linear component-wise interpolation was used to interpolate discrete field of the symmetric diffusion tensors characterizing fiber orientation from DT-MRI data to the centers of the generated tetrahedral elements. We assumed that the main eigen vector of the diffusion tensor shows the main fiber orientation $\hat{f}_{i}$ in the element.

We also assumed that electrical conductivity is constant in all directions perpendicular to the main fiber orientation. The conductivity along the fiber axes was taken to be $\sigma_{l}=1.2 \frac{\mathrm{mS}}{\mathrm{cm}}$ that was 1.5 times greater than across the fiber $\sigma_{t}$. Thus, orthogonal conductivity tensor was defined as $\bar{\Sigma}=\operatorname{diag}\left\{\sigma_{\mathrm{l}}, \sigma_{\mathrm{t}}, \sigma_{\mathrm{t}}\right\}$ and assigned to each tetrahedron according to the main fiber orientation $\hat{f}_{i}$ in this element by the tensor rotation: $\Sigma=M \bar{\Sigma} M^{T}$, where $\mathrm{M}$ is a rotational matrix based on $\hat{f}_{i}$. 
For the 2D model of the LV slice the conductivity tensor was projected from the tetrahedron vertices onto nearest neighbor triangle vertices $\tilde{\Sigma}=P(\mathbf{n}) \boldsymbol{\Sigma} \mathbf{P}(\mathbf{n})^{\mathbf{T}}$, where $\mathbf{n}$ is a normal vector to the slice, $\mathbf{I}$ is a unit matrix and $P(\mathbf{n})$ is given by the following formula:

$$
\begin{array}{r}
P(n)=I-(n \otimes n)= \\
\left(\begin{array}{ccc}
\left(1-n_{x}^{2}\right) & -n_{x} n_{y} & -n_{x} n_{z} \\
-n_{x} n_{y} & \left(1-n_{y}^{2}\right) & -n_{y} n_{z} \\
-n_{x} n_{z} & -n_{y} n_{z} & \left(1-n_{z}^{2}\right)
\end{array}\right)
\end{array}
$$

Then the conductivity tensor was interpolated from the triangle vertices to the triangle centers by the nearest neighbor interpolation.

\subsection{Electrophysiology models}

The monodomain model was used to describe the excitation wave propagation in the $2 \mathrm{D}$ and $3 \mathrm{D}$ models.

$$
\nabla \cdot\left(\boldsymbol{\Sigma} \nabla \mathbf{V}_{\mathbf{m}}\right)=\beta\left(C_{m} \frac{\partial V_{m}}{\partial t}+I_{\text {ion }}+I_{\text {stim }}\right),
$$

where $V_{m}$ is the transmembrane potential, $\Sigma$ is the conductivity tensor, $\beta$ is the surface-to-volume ratio, $C_{m}$ is the membrane capacitance and $I_{\text {ion }}$ is the sum of transmembrane ionic currents described by Luo-Rudy I model [8] and $I_{\text {stim }}$ is the stimulation current. Excitation was initiated from the entire subendocardial layer (Fig. 1A) or from the point at the subendocardium of the apex (Fig. 1B).

Unipolar pseudo-ECG was recorded using following formula:

$$
\phi(\mathbf{r})=\int_{\boldsymbol{\Omega}} \nabla \mathbf{V}_{\mathbf{m}} \cdot \nabla \frac{\mathbf{1}}{\mathbf{R}(\mathbf{p}, \mathbf{r})} \mathbf{d r},
$$

where $\Omega$ is the entire myocardium domain, $R$ is the distance between the radius vector $\mathbf{r}$ and coordinates of the electrode $\mathbf{p}$. The location of the electrodes are presented in (Fig. 1C). Each pseudo-ECG signal for each electrode was normalized to its maximal amplitude to compare shapes of the ECG waveforms between the 2D and 3D models.

The simulations were performed using open source Oxford Chaste software and home-made software developed in Python.

\section{Results}

We present below the results on the differences in of depolarization and repolarization characteristics between the 2D and 3D models when the entire subendocardial layer or a point at the subendocardium of the LV apex were paced.

\subsection{Subendocardial pacing}

The comparison of the QRS complex and T wave shapes between the models is shown in Fig. 2. For both models QRS complex had negative R-peaks at 2-9 $\mathrm{ms}$ at the
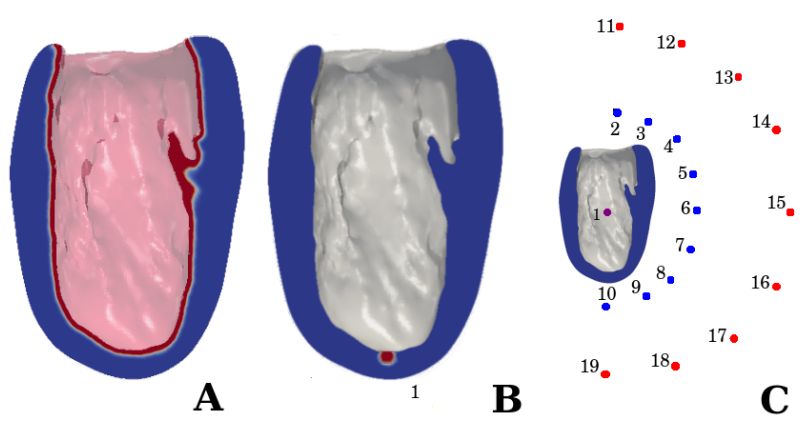

Figure 1. A: Subendocardial pacing. B: Apical pacing. $\mathrm{C}$ : The location of the electrodes for pseudo-ECG registration. Blue electrodes are located outside the LV at the distance of $45 \mathrm{~mm}$ from the LV center. Red electrodes are located at the distance of $90 \mathrm{~mm}$.

electrodes located close to the LV base $(2,11,12)$. For both models positive $\mathrm{R}$ peak and deep negative $\mathrm{S}$ wave were observed in the apical and in the central direction $(4,5,6,7,8,9,10,14,15,16,17,18,19)$. Only at electrodes 3 and 13 we found significant differences in the QRS configuration between the $2 \mathrm{D}$ and $3 \mathrm{D}$ models with negative $\mathrm{R}$ peak for the $3 \mathrm{D}$ but not for the 2D model. Other electrodes showed quite similar QRS configuration, however differences in the signal shapes with faster $\mathrm{R}$ wave onset in the 2D model were observed.

The comparison of the $\mathrm{T}$ wave between the models also revealed marked differences with a signal in the opposite direction at electrodes 9 and 19. Biphasic T-wave was observed at electrodes 9,19 in the 2D model but at the electrode 19 in the 3D model. Similar configuration was obtained at the other electrodes.

To analyze ECG wave shape differences observed between the 2D and 3D models we estimated activation and repolarization times as well as dispersion of repolarization and action potential duration at $90 \%$ repolarization $\left(\mathrm{APD}_{90}\right)$ for the 2D model of the longitudinal LV slice, 3D model of the whole LV and the LV slice processed from the $3 \mathrm{D}$ model with the same contour like of the $2 \mathrm{D}$ model. We found that differences between the models were within 3-4 $\mathrm{ms}$ as for estimation throughout the LV free wall and septum (Table 1) as for calculations for free wall/septum only (not shown). Further analysis of activation and repolarization maps showed regional differences between the models up to $5 \mathrm{~ms}$ for activation time (Fig. 3), $4 \mathrm{~ms}$ for repolarization time, $1 \mathrm{~ms}$ for repolarization dispersion and $3 \mathrm{~ms}$ for dispersion of $\mathrm{APD}_{90}$ (not shown). Additional analysis did not show significant differences in the propagation of the excitation wave. In the case of subendocardial pacing the excitation wave spread transmurally in both $2 \mathrm{D}$ and $3 \mathrm{D}$ models. 


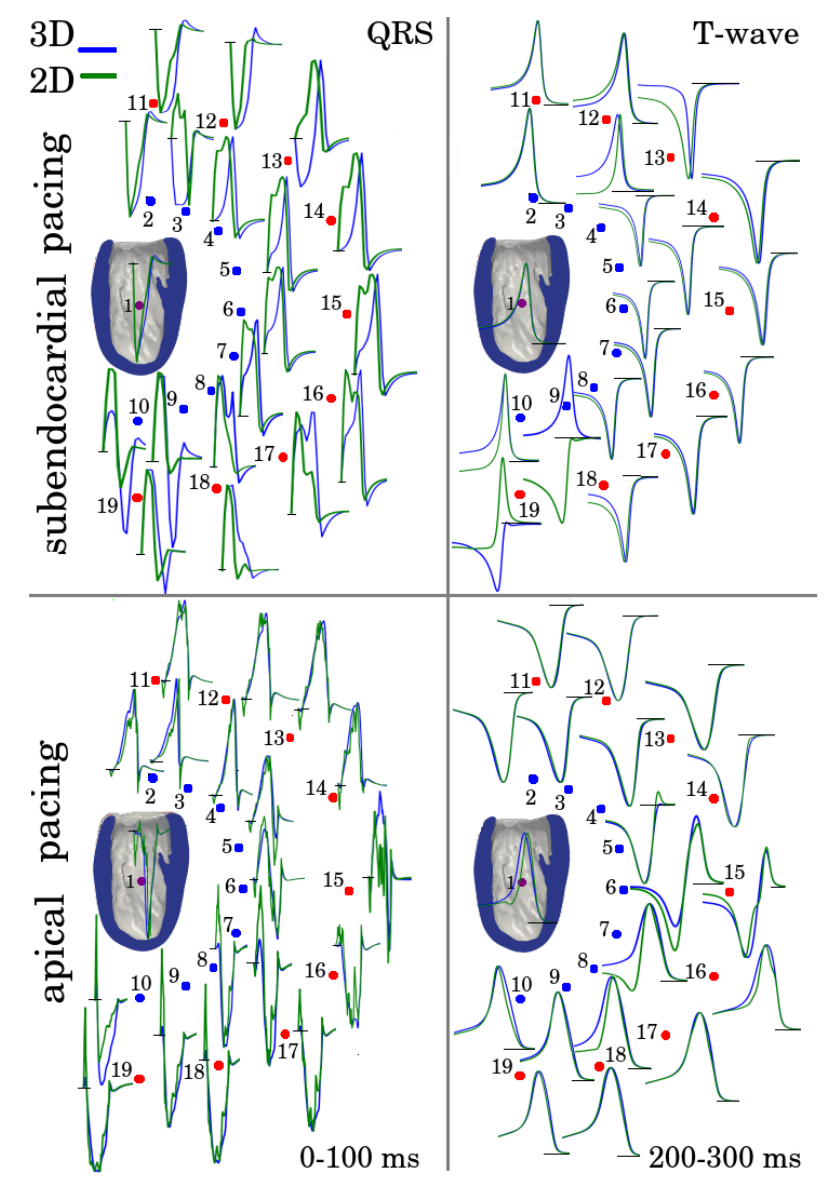

Figure 2. The comparison of the QRS complex and T wave shapes between the $2 \mathrm{D}$ model of the longitudinal ventricular slice and the $3 \mathrm{D}$ model of the whole $\mathrm{LV}$

\subsection{Apical Pacing}

The QRS complex and the T wave shapes for the apical pacing are shown in Fig. 2. Electrodes with positive R-peak were located in basal and central directions $(2,3,4,5,6,11,12,13,14,15)$. For these electrodes QRS complex shapes were perfectly matched between the $3 \mathrm{D}$ and 2D models excepting the electrode 15. Unlike subendocardial pacing QRS complex had negative $\mathrm{R}$ peaks at 9-12 $\mathrm{ms}$ at the electrodes located close to the LV apex $(7,8,9,10,16,17,18,19)$. The maximum differences between the 2D and 3D models were observed for these electrodes.

Biphasic T-wave was observed in the central region of the LV at electrodes 5,6,7,15 for the 2D model but at the electrodes 6,15 for the $3 \mathrm{D}$ model. Thus, the $\mathrm{T}$ wave at the electrodes 5,7 in the 3D model became biphasic in the 2D model. For other electrodes $\mathrm{T}$ wave amplitudes were similar for the 2D and 3D models.

As in the case of subendocardial pacing, the activation

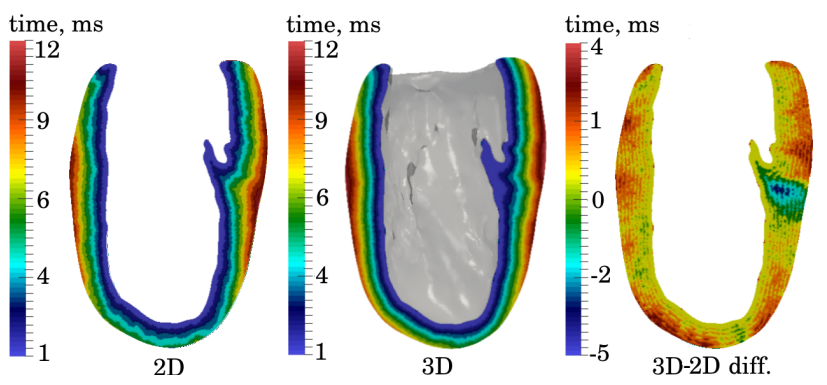

Figure 3. Activation maps of the 2D and 3D models and its differences in the case of subendocardial pacing.

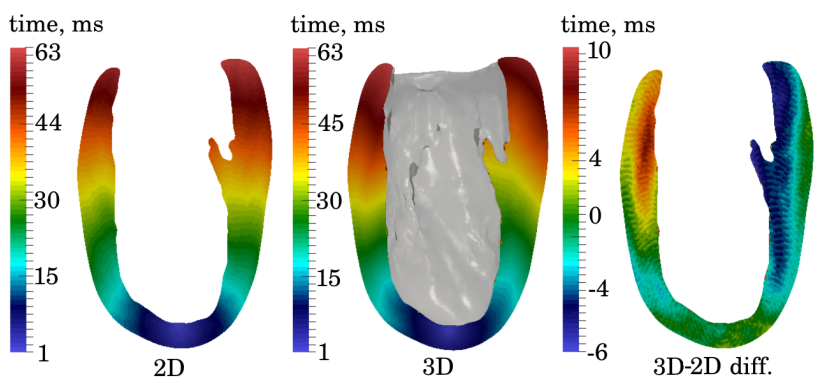

Figure 4. Activation maps of the $2 \mathrm{D}$ and $3 \mathrm{D}$ models and its differences in the case of apical pacing.

and repolarization times as well as dispersion of $\mathrm{APD}_{90}$ did not differ between the models more than 3-4 ms as for estimation throughout the LV free wall and septum (Table 1) as for calculations for free wall/septum only (not shown). However, analysis of activation and repolarization maps showed regional differences between the models up to $10 \mathrm{~ms}$ for activation time (Fig. 4), $10 \mathrm{~ms}$ for repolarization time, $2 \mathrm{~ms}$ for repolarization dispersion and $3 \mathrm{~ms}$ for dispersion of $\mathrm{APD}_{90}$ (not shown).

Additional analysis has shown that from 0 to $10 \mathrm{~ms}$, the excitation wave spread circumferentially in both models. After $10 \mathrm{~ms}$ significant differences in the wavefront shapes were observed. In the septum of the 2D model, excitation wavefront was convex and activated the subendocardial layer 1-3 ms faster then subepicardial one. In the septum of the 3D model the wavefront was concave and activated both borders almost simultaneously. In the free wall of the 2D model the wavefront was almost flat and perpendicular to both borders while in the free wall of the 3D model it was inclined and activated the subendocardial layer $1-4 \mathrm{~ms}$ faster then subepicardial one.

\subsection{Discussion and conclusions}

In this study we investigated the differences in electrophysiological characteristics between the 3D model of the LV and the 2D model of the longitudinal ventricular slice passing through the apex. The ECG wave shape compar- 
Table 1. The comparison of electrophysiological properties between the 2D model of the longitudinal LV slice, 3D model of the whole LV and the LV slice processed from the 3D model. Values are given in ms.

\begin{tabular}{|c|c|c|c|}
\hline & $2 \mathrm{D}$ & $3 \mathrm{D}$ & $2 \mathrm{D}$ from the $3 \mathrm{D}$ \\
\hline \multicolumn{4}{|c|}{ Subendocardial pacing } \\
\hline Activation time & 10 & 11 & 12 \\
\hline Repolarization time & 327 & 330 & 330 \\
\hline Repolarization dispersion & 20 & 21 & 21 \\
\hline Dispersion of $\mathrm{APD}_{90}$ & 11 & 10 & 8 \\
\hline \multicolumn{4}{|c|}{ Apical pacing } \\
\hline Activation time & 59 & 63 & 63 \\
\hline Repolarization time & 384 & 387 & 388 \\
\hline Repolarization dispersion & 69 & 70 & 71 \\
\hline Dispersion of $\mathrm{APD}_{90}$ & 11 & 12 & 14 \\
\hline
\end{tabular}

ison was performed using normalized signals because absolute values significantly differ due to a difference in myocardium mass between the 3D and 2D models.

We have shown that the electrophysiological processes in the ventricle and the longitudinal ventricular slice are quite similar, so we believe that cardiac slices can be used to evaluate global electrophysiological properties of the ventricles. We found that differences in the ECG wave shapes between the models may be dependent on the pacing region and a location of the ECG electrode. For subendocardial pacing the best matches of the signal of the $2 \mathrm{D}$ and 3D models were obtained at electrodes located close to the basal region. When subendocardium of the apex was paced pseudo-ECG was similar at the electrodes located close to the LV basal and apical regions.

To analyze ECG signal differences we estimated activation and repolarization times as well as dispersion of repolarization and $\mathrm{APD}_{90}$ for the $2 \mathrm{D}$ model of the longitudinal LV slice, 3D model of the whole LV and the LV slice processed from the 3D model. We found that the values in the models did not differ more than 3-4 ms. These results explain general similarities in the ECG wave peak times between the 2D and 3D models. However, analysis of the activation and repolarization maps showed regional differences between the models underlying the observed ECG wave shape differences. These regional differences may be explained by different patterns of the excitation wave propagation in the models especially in the case of apical pacing. In this case longer excitation wave propagation led to more pronounced changes in wavefront shape in comparison with subendocardial pacing. Also, for the apical pacing, the myocardial anisotropy of the free wall and the septum affected the excitation waves in different ways. We suggest that fiber orientation patterns of the 2D and 3D models may affect excitation wave propagation resulting in the differences in the ECG wave shape despite general sim- ilarities in depolarization and repolarization between the 2D and 3D models.

\section{Acknowledgements}

This work was supported by IIF UrB RAS theme \#AAAA-A18-118020590031-8, RFE Government Act \#211 of March 16, 2013, the Program of the Presidium RAS \#27 and RFBR (\#16-31-60015, 18-31-00401).

\section{References}

[1] De Boer TP, Camelliti P, Ravens U, Kohl P. Myocardial tissue slices: organotypic pseudo-2d models for cardiac research \& development. Future Cardiology 2009;5(5):425430.

[2] Wang K, Lee P, Mirams GR, Sarathchandra P, Borg TK, Gavaghan DJ, Kohl P, Bollensdorff C. Cardiac tissue slices: preparation, handling, and successful optical mapping. American Journal of Physiology Heart and Circulatory Physiology 2015;308(9):H1112-H1125.

[3] Borile G, De Mauro C, Urbani A, Alfieri D, Pavone FS, Mongillo M. Multispot multiphoton $\mathrm{Ca}^{2+}$ imaging in acute myocardial slices. Journal of Biomedical Optics 2014; 20(5):051016.

[4] Pillekamp F, Halbach M, Reppel M, Rubenchyk O, Pfannkuche K, Xi JY, Bloch W, Sreeram N, Brockmeier K, Hescheler J. Neonatal murine heart slices. a robust model to study ventricular isometric contractions. Cellular Physiology and Biochemistry 2007;20(6):837-846.

[5] Yamashita D, Kohzuki H, Kitagawa Y, Nakashima T, Kikuta A, Takaki M. $\mathrm{O}_{2}$ consumption of mechanically unloaded contractions of mouse left ventricular myocardial slices. American Journal of Physiology Heart and Circulatory Physiology 2004;287(1):H54-H62.

[6] Parrish A, Dorr R, Gandolfi A, Brendel K. Adult rat myocardial slices: A tool for studies of comparative cardiotoxicity. Toxicology in Vitro 1994;8(6):1233-1237.

[7] Camelliti P, Al-Saud SA, Smolenski RT, Al-Ayoubi S, Bussek A, Wettwer E, Banner NR, Bowles CT, Yacoub MH, Terracciano CM. Adult human heart slices are a multicellular system suitable for electrophysiological and pharmacological studies. Journal of Molecular and Cellular Cardiology 2011;51(3):390-398.

[8] Luo Ch, Rudy Y. A model of the ventricular cardiac action potential. Depolarization, repolarization, and their interaction. Circulation Research 1991;68(6):1501-1526.

Address for correspondence:

Anastasia E. Bazhutina

Ural Federal University,

Mira 19, Ekaterinburg 620002, Russia.

E-mail: banas49@mail.ru 